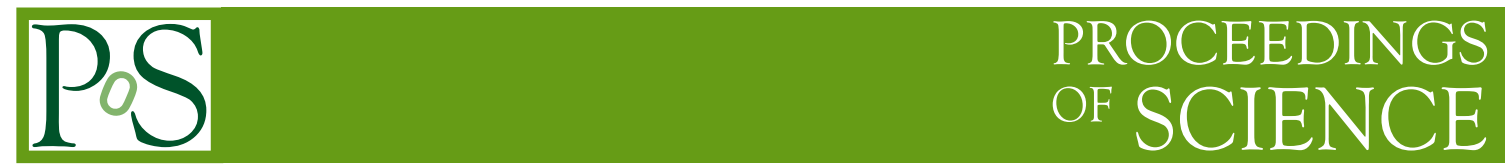

\title{
New algorithms for finite density QCD
}

\author{
Dénes Sexty* \\ ITP, University of Heidelberg \\ Department of Physics, University of Wuppertal \\ E-mail: sexty.denesegmail.com
}

Recent progress of the complex Langevin method and the Lefschetz thimble in connection with the sign problem is reviewed. These methods rely on the complexification of the original field manifold and they allow direct simulations of theories with non-real measures. Similarities and differences of the two approaches are pointed out. Results using the complex Langevin method, which allows simulations to evade the sign problem in full QCD, are presented. Promising results of the thimble approach for non-gauge theories are also discussed.

The 32nd International Symposium on Lattice Field Theory,

23-28 June, 2014

Columbia University New York, $N Y$

${ }^{*}$ I gratefully thank my collaborators G. Aarts, F. Attanasio, L. Bongiovanni, Sz. Borsányi, Z. Fodor, B. Jäger, S.D. Katz, E. Seiler and I.-O. Stamatescu. 


\section{Introduction}

Investigations of the non-zero density phases of QCD are hampered by the sign problem. The theory is defined with the path integral

$$
Z=\int D U e^{-S_{g}[U]} \operatorname{Det} M(U, \mu)
$$

with the gauge action $S_{g}[U]$ and the determinant of the fermion matrix $M(U, \mu)$. For nonzero chemical potential $\mu$, the determinant becomes complex, thus importance sampling based simulation methods are not applicable. For small chemical potentials, the ensemble is close to the $\mu=0$ ensemble, thus there are various options to evade the sign problem, such as reweighting, Taylor expansion, analytic continuation from imaginary chemical potentials, etc. (see the reviews $[1,2]$ and citations therein). Most of these methods work by simulating in an ensemble with a positive measure, where naive simulations can be performed, and then trying to recover the original, non-positive ensemble with a clever choice of the observables, or some kind of expansion or extrapolation. These methods fail to recover the original ensemble if it is 'too far' from the positive ensemble, usually they are limited to the region $\mu / T<\mathrm{O}(1)$.

In this paper I'm going to review recent progress in two direct simulation methods, which allow simulations of the non-positive ensemble we are interested in directly. They are both based on analytical continuation: they expand the field manifold into the complex plane.

The first of these methods is the complex Langevin method [3]. It is the straightforward extension of the idea of stochastic quantisation [4] to complex actions. After some initial excitement in the 80's, interest in the method has largely disappeared, as it was noticed that the method in some cases gives wrong results, even though in other cases it works in spite of a hard sign problem. In recent years, renewed interest in the method has produced studies with many important new results. Our theoretical understanding of the failures and successes of the method has improved substantially, with some details still missing (see Sec. 2 for details). Based on this understanding, an important ingredient for the simulations of gauge theories called gauge cooling has been introduced [5]. This procedure removes an inherent instability of the naive complex Langevin process of gauge theories, connected to the complexification of the gauge degrees of freedom. Gauge cooling was used to solve the sign problem in HDQCD in which the spatial fermionic hoppings are dropped [5] (see also in Sec. 6), and it has been extended also to full QCD with light quarks [6].

The complex Langevin method can of course be used for various other problems with complex actions such as the problem of real time evolution [7], as well as gauge theories with a $\Theta$-term [8].

The second method for direct simulations is the Lefschetz thimble. The idea extends the saddle point integration to a non-perturbative tool which is equivalent to the usual path integral. The theory defined on the path through the saddle point (the 'Lefschetz thimble') is much better behaved from the point of view of the sign problem [9]. After the initial proposal the idea was quickly realized numerically for various models with promising results, see Sec. 5 for references.

This article is organized as follows: in Sec. 2 the complex Langevin method is briefly described. In Sec. 3 an overview of the theoretical foundation of the Lefschetz thimble is given. In Sec. 4, both methods are applied to a simple toy model to illustrate how they evade the sign problem. In Sec. 5, recent numerical results using the Lefschetz thimble are briefly reviewed. In Sec. 6 , 
I discuss results of the HDQCD approximation. In Sec. 7, the $\kappa$ - and $\kappa_{s}$-expansions are described. In Sec. 8, results of the application of CLE to full QCD are described. Finally Sec. 9 concludes.

\section{Complex Langevin equation}

The Complex Langevin method is based on the analytical continuation of a Langevin process to a complexified manifold. Let's consider an action of one real variable $S(x)$ for demonstration. The real Langevin equation is written as $d x(\tau) / d \tau=-\partial_{x} S(x)+\eta(\tau)$, with the Langevin time $\tau$ and the noise term $\eta(\tau)$ satisfying $\langle\eta(\tau)\rangle=0$ and $\left\langle\eta(\tau) \eta\left(\tau^{\prime}\right)\right\rangle=2 \delta\left(\tau-\tau^{\prime}\right)$. If the action is complex, the drift term $-\partial_{x} S(x)$ will be complex as well, and the field $x \rightarrow z=x+i y$ will develop an imaginary part. We define the drift for a complex field with analytical continuation to arrive at the complex Langevin equation (CLE)

$$
\frac{d z}{d \tau}=-\frac{\partial S(z)}{\partial z}+\eta(\tau)
$$

The noise term can also be complexified but for practical simulations it is better to keep it real [10]. The complex Langevin process thus enlarges the manifold of the variables to a non-compact group, in the case of lattice gauge theories the $\mathrm{SU}(\mathrm{N})$ link variables become $\mathrm{SL}(\mathrm{N}, \mathbb{C})$, see below. The observables of the original theory are recovered using the principle of analytic continuation

$$
\langle F\rangle=\frac{1}{Z} \int d x e^{-S(x)} F(x)=\int d x d y P(x, y) F(x+i y)
$$

for the observable $F(z)$, where $P(x, y)$ is the real distribution function of the complexified variable $z=x+i y$ of the equilibrated complex Langevin process. Note that this prescription is equivalent to averaging the observable for the configurations of an equilibrated complex Langevin process

$$
\langle F\rangle=\lim _{T \rightarrow \infty} \frac{1}{T} \int_{0}^{T} d \tau F(z(\tau)),
$$

where $z(\tau)$ is the solution of (2.1).

The Complex Langevin method is known to deliver wrong results in some cases, but our understanding of this issue has improved recently. One can prove that the Complex Langevin approach delivers correct results, if some conditions are satisfied: the action and the observables should be holomorphic functions, and the distribution of the variables in the complexified manifold should decay sufficiently fast [10]. The failure of the method is usually the consequence of the violation of the second condition: slow decay of distributions. In the case of non-zero density QCD, the first of these conditions is violated, the effective action we want to simulate is $S_{\text {eff }}=$ $S_{g}-\ln$ Det $M$, where the logarithm has a branch cut. In consequence the drift term calculated from the action has poles where the measure $e^{-S_{g}} \operatorname{Det} M$ vanishes. The theoretical understanding of this issue is still limited. The logarithm in the action can lead to problems in some cases [11, 12], but there are also cases when the presence of a logarithm causes no harm, as is typically the case when the gauge group is parametrised with the help of a reduced Haar measure [13, 14]. In the case of QCD, we have evidence that this issue has so far has not shown up: comparison with reweighting for small chemical potentials shows that CLE gives correct results [15], the results of full QCD are 
reproduced with an approach ( $\kappa$-expansion) where the action is holomorphic [16] (see also Sec. 7), but it remains to be seen whether low temperatures in particular are affected.

The Complex Langevin approach can be easily generalized to a curved manifold such as the $\mathrm{SU}(\mathrm{N})$ group. The discretised update for one variable $U \in \mathrm{SU}(\mathrm{N})$ reads (with straightforward generalization to a lattice system):

$$
U(\tau+\varepsilon)=R(\tau) U(\tau)
$$

where the update is defined as $R(\tau)=\exp \left(i \lambda_{a}\left(K_{a}+\eta_{a} \sqrt{\varepsilon}\right)\right)$, with the generators $\lambda_{a}$, and noise terms $\eta_{a}$ satisfying $\left\langle\eta_{a}\right\rangle=0,\left\langle\eta_{a} \eta_{b}\right\rangle=2 \delta_{a b}$. The drift term is calculated from the action $S(U)$ as $K_{a}=-D_{a} S(U)$ with the left derivative $D_{a}$.

The complexification of the degrees of freedom for theories with local symmetry enlarges also the gauge degrees of freedom. The complex Langevin process then tries to explore these noncompact gauge orbits which results in large fluctuations and slow decay of field distributions, and thus incorrect results. This undesirable behavior can be countered with a procedure called gauge cooling $[5,17]$. The 'distance' of the field configuration in the enlarged SL(N,C) field space from the original $\mathrm{SU}(\mathrm{N})$ manifold can be quantified by the unitarity norm:

$$
\operatorname{Tr}\left(U^{+} U-1\right)^{2} \geq 0 .
$$

The gauge cooling procedure reduces this quantity using the gauge symmetry of the link variable $U_{v, x}$ in direction $v$ at position $x$

$$
U_{v, x} \rightarrow \Omega(x) U_{v, x} \Omega\left(x+a_{v}\right),
$$

where $\Omega(x) \in \mathrm{SL}(\mathrm{N}, \mathbb{C})$ is chosen by the requirement that the average of the unitarity norm (2.5) over the lattice should be minimal. In practice we choose $\Omega(x)$ to be proportional to the gradient of the unitarity norm, and apply several gauge cooling steps after each dynamical update of the form (2.4). For more advanced gauge cooling algorithms, see Ref. [17].

The empirical observation is that the gauge cooling procedure is effective as long as the $\beta$ parameter of the gauge action is not too small. The minimal usable value, $\beta_{\min }$, for which the results of the Complex Langevin simulation agree within errors with other approaches (where available, such as reweighting) appears to depend very weakly on the lattice size and the chemical potential [18]. Whether a particular $\beta$ value is usable can also be judged from the CLE simulation itself: for too small $\beta$ values the distribution of the variables develops a slowly decaying tail ('skirt'). In theories with different fermionic content the actual value of $\beta_{\min }$ might be different, but it turns out this is the usual renormalization effect: $\beta_{\min }$ corresponds to the maximal usable lattice spacing of $a_{\max } \approx 0.1-0.2 \mathrm{fm}$, roughly the same value independently of the numbers of flavors and their masses. This means that the continuum limit of the theory can be reached in the safe region where the method is well controlled, but there is a drawback that large lattices have to be used for small temperatures.

\section{Lefschetz thimble}

We are interested in calculating averages in a theory defined by the partition function

$$
Z=\int d x M(x)=\int \exp (-S(x)) D(x) d x
$$


where the measure $M(x)$ is typically written in the form $M(x)=\exp (-S(x)) D(x)$ as indicated, with the action $S(x)$ and $D(x)$ which can be a Haar measure (or some other Jacobian), as well as a fermionic determinant, and the integration goes over the real axis. Let's first notice that we don't necessarily have to perform the integration in our theory for real $x$ fields.

$$
\int_{-\infty}^{\infty} d x M(x) O(x)=\int_{C} d z M(z) O(z)=\int_{-\infty}^{\infty} d t J(t) M(z(t)) O(z(t))
$$

where $C$ is some contour in the complex plane parametrized as $z(t)$, the deformation of the real axis, and $J(t)=d z / d t$ is the Jacobian corresponding to this variable transformation. This equality is valid as long as the measure $M(z)$ and the observable $O(z)$ don't have any singularities which the integration path crosses as it is deformed. In the theories we are interested in, this is satisfied.

Typically the measure $M(x)$ is real and positive, so from the point of view of numerical simulations there is no reason to deform the contour into the complex plane. In the cases where $M(x)$ is complex it's worth deforming the contour, if on the new contour the real part of the action is more peaked, therefore sampling is more efficient, and/or the imaginary part has less fluctuations, therefore reweighting is easier. Fortunately these two requirements specify the same curves (manifolds in higher dimensions): the Lefschetz thimbles.

The stable (instable) Lefschetz thimbles are defined as the paths of the steepest descent (ascent) of the real part of the action on the complex plane, starting from a critical point, where $\partial_{z} S(z)=$ 0 . The steepest descent (ascent) of the real part and the constancy of the imaginary part specify the same curves for a holomorphic measure, as can be seen by examining the Cauchy-Riemann relations. If we choose the stable thimbles as the domain of the integration, the real part of the action is squeezed closest to the peak, as the path follows the steepest descent. The imaginary part of the action $S_{I}$ is constant on the thimbles, therefore the factor $\exp \left(-i S_{I}\right)$ it can be pulled out of the integral. The sign problem does not completely disappear, it is reintroduced by the factor $J(t)$, which is in general complex. This is called the residual sign problem, and it reappears as long as the deformed contour has curvature (which is equivalent to the model being non-Gaussian). In the case where more than one thimble contributes, a global sign problem also appears as one sums contributions from different thimbles. The residual sign problem is usually much milder than the original one, but its dependence on the volume is probably still exponential, preventing the usage of large volumes, but allowing to draw some conclusions about the infinite volume theory.

\section{Lefschetz thimble and Complex Langevin solution of a toy model}

First a simple toy model is examined with both methods to illustrate how they evade the sign problem. The action we are interested in is

$$
S(x)=\sigma x^{2}+i \lambda x
$$

This model is Gaussian, therefore the exact solution can be calculated analytically. Naive simulations would take the real part of the action, and reweight the imaginary part. Already for this simple model this can be very challenging, with lots of cancellations required (see left panel of Fig. 1) to get the exact result. 

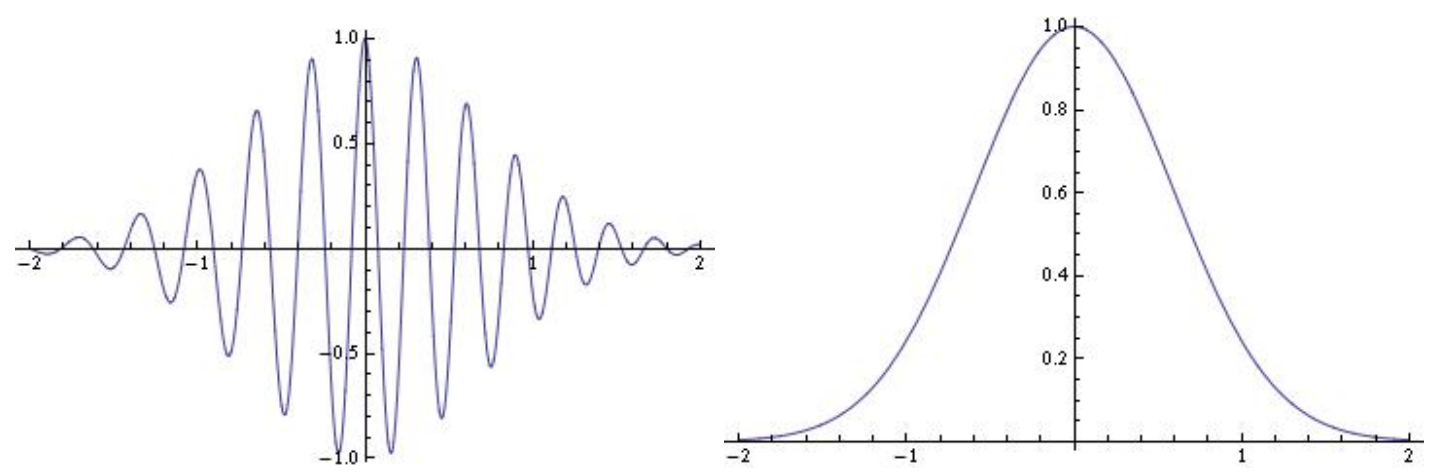

Figure 1: The measure of the Gaussian toy model defined in eq. (4.1) on the real axis (left) and on the stable thimble (right) using $\sigma=1+i$ and $\lambda=20$.

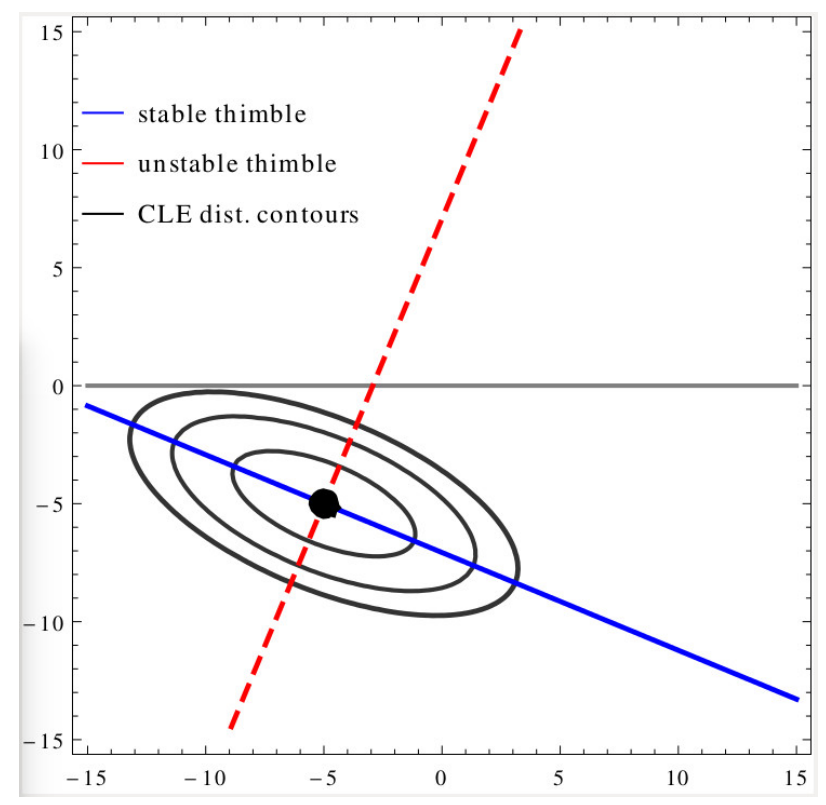

Figure 2: The complexification of the Gaussian toy model defined in eq. (4.1). The stable (unstable) thimble is given by the blue (red, dashed) line. The black ellipses signify the equal probability levels of the equilibrium distribution of the complex Langevin process.

This action has one critical point at $z_{c}=-i \lambda / 2 \sigma$. The thimbles attached to this point are straight lines (see Fig. 2), thus the residual phase problem is absent (this simplification happens only for non-interacting theories). The measure on the thimble is well peaked and is easily sampled with a Monte-Carlo process of choice, see right panel of Fig. 1.

In the complex Langevin approach we set up the complexified Langevin equation

$$
\frac{d z}{d \tau}=-2 \sigma z-i \lambda+\eta(\tau)
$$

In this simple case the probability distribution of $z$ on the complex plane can be given analytically: in the equilibrium it is given by the Gaussian distribution, the solution of the Fokker-Planck 
equation as displayed in Fig. 2. Using this distribution in eq. (2.2) one gets the correct averages.

Note that the distribution gained with the complex Langevin process is peaked around the critical point of the action, where also in the thimble approach the most dominant contributions are located. This similarity carries over to interacting theories as well, first noted by Aarts [19], but there are differences as well. The distribution of the complex Langevin process is usually "near" to a thimble of the theory, but it is not necessarily peaked near the peak of the measure on the thimble. The relationship is also complicated by instable fixed points in the complex Langevin dynamics, and different behavior near a zero of the measure [20].

\section{Numerical implementations of the Lefschetz thimble}

In this section a short overview is given of the status of the implementation of the idea of the Lefschetz-thimbles in actual numerical calculations. These implementations have several difficulties to overcome: One has to come up with a way to sample the thimble with the weight $\exp \left(-S_{R}\right)$, i.e. generate configurations that are solutions of the thimble equation (written for a scalar field $\left.\phi=\phi_{R}+i \phi_{I}\right)$

$$
\begin{aligned}
\frac{d}{d \tau} \phi_{R}(\tau) & =-\frac{\delta S_{R}[\phi(\tau)]}{\delta \phi_{R}} \\
\frac{d}{d \tau} \phi_{I}(\tau) & =-\frac{\delta S_{R}[\phi(\tau)]}{\delta \phi_{I}}
\end{aligned}
$$

for some initial $\tau$ and critical point such that $\phi(\tau=\infty)=\phi_{c}$, and measure the residual phase for these configurations (which in general is a determinant of a large matrix, efficient calculation methods are needed [21]). The theory is still not free of the sign problem: the residual phase is than taken into account with reweighting, as it should present a mild sign problem. For field theories it is expected that only one critical point (corresponding to the global minimum of the action) needs to be taken into account [9], but in toy models with only a few degrees of freedom all critical points and their thimbles can be important [20].

With the help of a mapping between a Gaussian thimble and the curved thimble one can perform random Metropolis sampling as has been demonstrated for toy models in [22]. One possible way to sample the thimble for a field theory is using a real Langevin equation [9]

$$
\begin{aligned}
\frac{d}{d \tau} \phi_{R}(\tau) & =-\frac{\delta S_{R}[\phi(\tau)]}{\delta \phi_{R}}+\eta_{R} \\
\frac{d}{d \tau} \phi_{I}(\tau) & =-\frac{\delta S_{R}[\phi(\tau)]}{\delta \phi_{I}}+\eta_{I} .
\end{aligned}
$$

This equation is the same as the steepest descent equation defining the thimble (5.1) for zero $\eta$ noise terms. One only has to make sure that the noise does not drive the system off the thimble, i.e. it has to be tangential to it. This is achieved by transporting the noise back to the critical point to be constrained to the tangential of the thimble (which is simply the space spanned by positive eigenvalues of the Hessian of the action at $\phi_{c}$ ), and then transporting it back to $\phi$. This procedure has been applied to the $\phi^{4}$ theory [23] and to the Hubbard model away from half filling in [24] with promising results. 
In an impressive paper [25] the Hybrid Monte Carlo algorithm was implemented for the $\phi^{4}$ scalar theory with a chemical potential, where they could compare to available results using CLE [26]. The authors studied the residual sign problem and saw that it is indeed much milder than the original sign problem of the theory in the naive formulation.

Further sampling algorithms were discussed also in [27].

In summary the application of the Lefschetz thimble to simulations of field theories and toy models has yielded very encouraging results so far, but it is yet to be tried on a theory closer to full QCD (or on a theory with local symmetries).

\section{CLE treatment of the HDQCD model}

In this section the first order of a systematic approximation to QCD called HDQCD is described. Consider the Wilson fermion matrix

$$
M(x, y)=1-\sum_{v=1}^{4} \kappa_{v}\left(\left(1-\gamma_{v}\right) \exp \left(\delta_{v 4} \mu\right) U_{v}(x) \delta_{y, x+a_{v}}+\left(1+\gamma_{v}\right) \exp \left(-\delta_{v 4} \mu\right) U_{v}^{-1}(y) \delta_{y, x-a_{v}}\right)(6.1)
$$

with the hopping parameters $\kappa_{v}$ (the spatial hopping $\kappa_{s}=\kappa_{1}=\kappa_{2}=\kappa_{3}$ ) and Euclidean Gammamatrices $\gamma_{v}$. We also use the notation

$$
M=1-\kappa Q=1-\kappa_{s} S-R,
$$

where $Q$ is the hopping matrix (it only contains terms connecting neighboring lattice sites), and $S(R)$ is the spatial (temporal) hopping matrix. The HDQCD theory corresponds to setting $\kappa_{s}=0$, which can be justified by considering the limit $\kappa \rightarrow 0, \mu \rightarrow \infty, \kappa e^{\mu}=$ const.. This approximation can also be considered as the leading order (LO) of a systematic $\kappa_{s}$-expansion, to be described in detail in Sec. 7. In HDQCD, the fermion determinant simplifies considerably:

$$
\operatorname{det} M_{\mathrm{LO}}=\prod_{\mathbf{x}} \operatorname{det}\left(1+C \mathscr{P}_{\mathbf{x}}\right)^{2} \operatorname{det}\left(1+C^{\prime} \mathscr{P}_{\mathbf{x}}^{-1}\right)^{2},
$$

where $C(\mu)=\left(2 \kappa e^{\mu}\right)^{N_{\tau}}$ and $C^{\prime}(\mu)=C(-\mu)$, and $\mathscr{P}_{\mathbf{x}}$ is the Polyakov loop. The second factor has no contributions to the physics at large $\mu$, but it restores the usual symmetry of the fermion determinant $\operatorname{Det} M(\mu)=\operatorname{Det} M\left(-\mu^{*}\right)^{*}$.

This model has been investigated using the CLE and gauge cooling in Ref. [5] (for a study using reweighting, see [28]). In Fig. 3 the behavior of the density $n=(T / V)\langle\partial \ln Z / \partial \mu\rangle$ (with $T$ temperature and $V$ spatial volume) and the average phase factor, the measure of the severeness of the sign problem, defined as

$$
\left\langle e^{2 i \varphi}\right\rangle=\left\langle\frac{\operatorname{Det} M(\mu)}{\operatorname{Det} M(-\mu)}\right\rangle
$$

is shown. The average phase factor shows that for intermediate chemical potentials the sign problem is hard, nevertheless the complex Langevin process is well behaved in contrast to e.g. reweighting.

The small chemical potential behavior resembles the low temperature 'Silver blaze' phenomenon [29]: The chemical potential cannot change the behavior of the system until it is large 


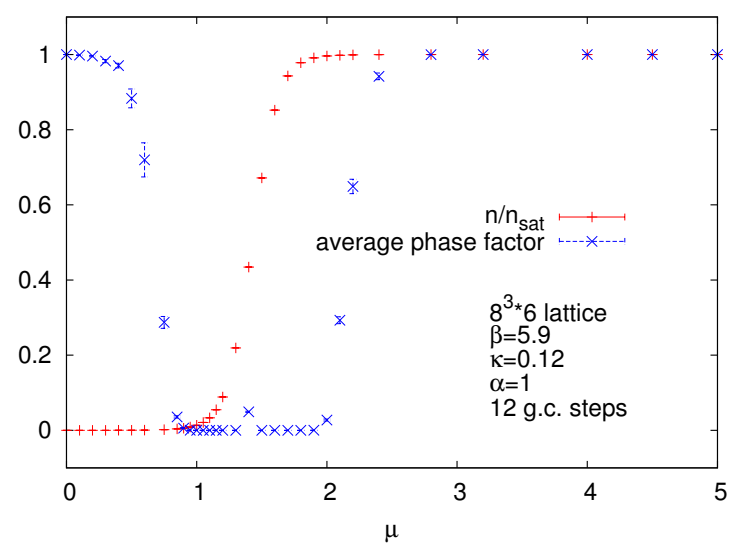

Figure 3: The density and the sign average in the HDQCD model at a fixed temperature as a function of the chemical potential.

enough to create fermionic excitations. One observes also the phenomenon of saturation: as the chemical potential is increased, the density of fermions can not grow past the value $n_{\text {sat }}$ which is realized when all states on the lattice are occupied. Deep in the saturation state the system behaves as a pure gauge theory, which can be interpreted as an 'inverse Silver Blaze' behavior: if there is not enough thermal energy to create a hole in the fermionic sea, the system will behave as a theory without fermions.

Comparison with reweighting and the investigation of distributions shows that the gauge cooling is no longer effective below $\beta_{\min } \approx 5.6$. Nevertheless one can go down to the confined phase by increasing the lattice size in the safe $\beta>\beta_{\min }$ region. This allows the determination of the whole phase diagram of the theory on the $\mu-T$ plane [30]. The dropping of the spatial hoppings has the consequence that the phase structure of this approximation is not as rich as the phase structure of full QCD. Recently we proposed 'closing the gap' with a systematic expansion in the $\kappa_{s}$ parameter, see in Sec. 7 and [16].

\section{7. $\kappa$ - and $\kappa_{s}$-expansion}

As discussed in Sec. 6, dropping the spatial fermionic hoppings simplifies the determinant such that the numerical treatment of the system becomes cheap. Accordingly, the physics of the model becomes also simple when compared to the richness of full-QCD. To recover some of the physics while keeping the numerical cost cheap, we propose to extend the leading order (LO) HDQCD simulations systematically with corrections of the order $\kappa_{s}^{n}$ [16]. We define two slightly different expansions: the $\kappa$-expansion expands the fermionic determinant as

$$
\operatorname{det} M=\operatorname{det}(1-\kappa Q)=\exp \sum_{n=1}^{\infty}-\frac{\kappa^{n}}{n} \operatorname{Tr} Q^{n},
$$

with straightforward generalization for more than one flavors. The hopping matrix $Q$ connects neighboring sites, therefore in the trace only even powers contribute. The fermionic observables 
are calculated similarly, using their definition on the expanded form, e.g. the fermionic density $\langle n\rangle=(T / V) \partial \ln Z / \partial \mu$.

The $\kappa_{s}$-expansion is defined by the identity

$$
\operatorname{det} M=\operatorname{det}(1-R)\left(1-\frac{1}{1-R} \kappa_{s} S\right)=\operatorname{det}(1-R) \exp \sum_{n=1}^{\infty}-\frac{\kappa_{s}^{n}}{n} \operatorname{Tr}\left(\frac{1}{1-R} S\right)^{n} .
$$

Since $S$ contains spatial hoppings, again only even powers contribute. The determinant (and the inverse) of the temporal part can be calculated analytically, and it gives back the HDQCD model in eq. (6.3).

These expansions can be implemented very efficiently in the complex Langevin simulations. The contribution to the drift of the $\kappa$-expansion (for a single flavor) is given by the trace

$$
K_{x v a}=-\sum_{n=1}^{\infty} \kappa^{n} \operatorname{Tr}\left(Q^{n-1} D_{x v a} Q\right)
$$

In the $\kappa_{s}$-expansion, the contribution from the LO temporal determinant in Eq. (7.2) given by the drift at LO [31]. The second factor contributes with the traces

$$
\begin{aligned}
K_{x i a} & =-\sum_{n=1}^{\infty} \kappa_{s}^{n} \operatorname{Tr}\left(\frac{1}{1-R}\left(D_{x i a} S\right)\left[\frac{1}{1-R} S\right]^{n-1}\right), \\
K_{x 4 a} & =-\sum_{n=1}^{\infty} \kappa_{s}^{n} \operatorname{Tr}\left(\frac{1}{1-R}\left(D_{x 4 a} R\right)\left[\frac{1}{1-R} S\right]^{n}\right),
\end{aligned}
$$

for spatial and temporal links, correspondingly. These traces are estimated using a noisy vector, i.e. by choosing a Gaussian random vector $\alpha_{i}$ (here $i$ represents space-time, color and Dirac indices) with $\left\langle\alpha_{i}\right\rangle=0,\left\langle\alpha_{i}^{*} \alpha_{j}\right\rangle=\delta_{i j}$, and then constructing the drift as

$$
K_{x v a}=\left\langle\alpha^{*}\left(D_{x v a} Q\right) s\right\rangle, \quad s=-\sum_{n} \kappa^{n} Q^{n-1} \alpha,
$$

for the $\kappa$-expansion. The dominant numerical cost of including fermions, with corrections up to $\kappa^{n}$, is thus $n-1$ multiplications with the sparse matrix $Q$. This should be contrasted with the implementation of full QCD, where the fermionic matrix is usually inverted with a conjugate gradient algorithm, which requires a multiplication per iteration until convergence. For the $\kappa_{s}$ expansion the drift is computed similarly, with additional multiplications of the temporally dense but spatially diagonal matrix $(1-R)^{-1}$, as required.

The convergence of the expansions defined above is illustrated on Fig. 4. Note that the procedure outlined above allows calculation of the $\kappa$ - and $\kappa_{s}$-expansion to very high orders, where convergence can be checked explicitly. Earlier studies used the loop expansion where higher orders become prohibitively hard, so only $\kappa^{2}$ corrections were calculated $[32,33,34,35,28]$ in connection with the full gauge action. Also introducing a strong coupling expansion for the gauge action allows calculations up to the $\kappa^{4}$ order [36, 37, 38, 39, 40].

The $\kappa$ - and $\kappa_{s}$-expansions have different merits: the $\kappa$-expansion can be calculated cheaply, and is free of the problem of the poles (as it is written in the exponent), but convergence properties at high chemical potential are not good. The $\kappa_{s}$-expansion has better convergence properties at high 

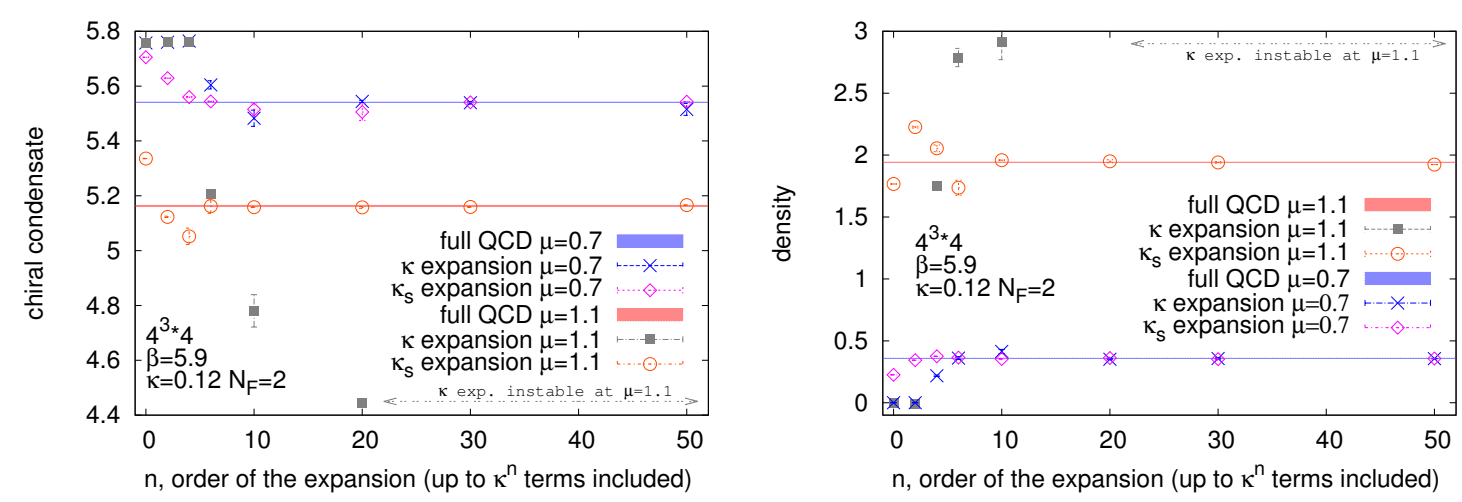

Figure 4: The results of the $\kappa-$ and $\kappa_{s}$-expansions as a function of the order of corrections included and the full QCD results.

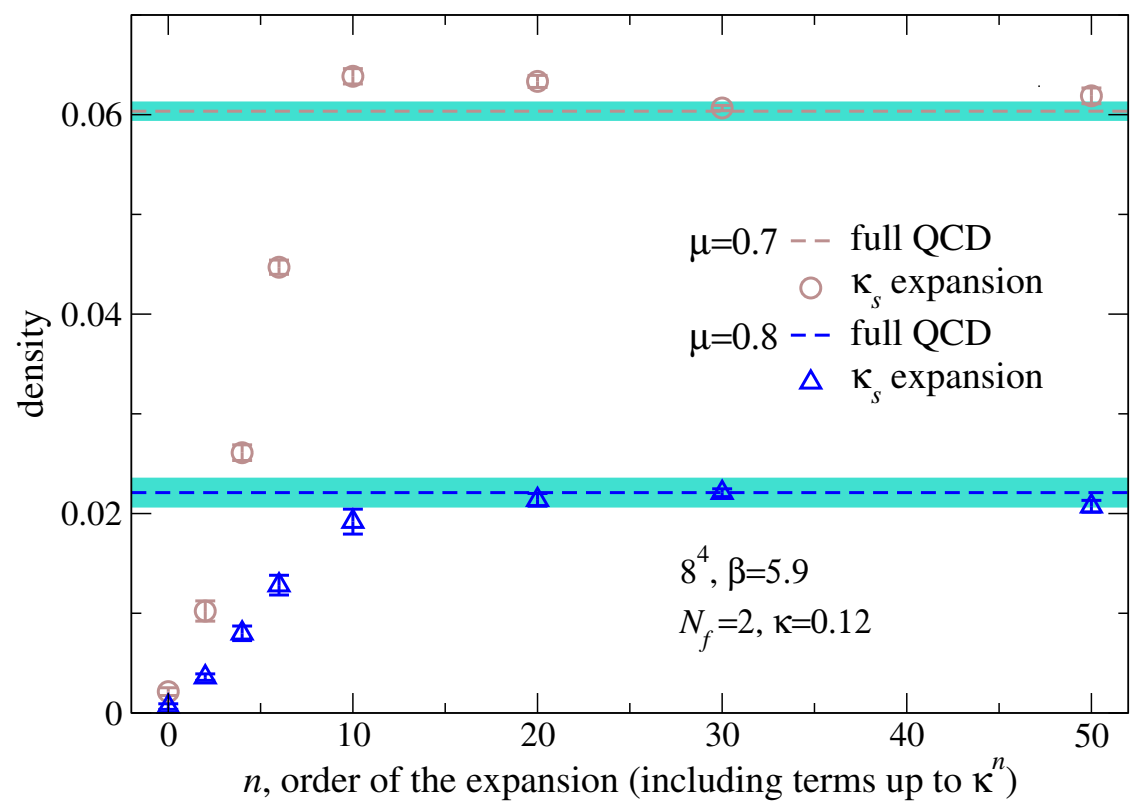

Figure 5: The density as a function of the order of corrections and the full QCD results on a $8^{4}$ lattice at two different chemical potentials.

$\mu$, but it is slightly more expensive, as the temporal part of the determinant is taken into account analytically, using a logarithm in the action. The convergence radius of the expansions does not seem to depend on the (temporal or spatial) size of the lattice, see in Fig. 5. In this case we tested the theory below the deconfinement transition, for two different $\mu$ values, and the rapid change in the density indicates the presence of the onset transition.

The fact that the expansion converges (for a certain parameter range) to the results of full QCD validates both theories: the expansion is used within its convergence radius and the full theory is apparently not influenced by the non-holomorphicity of the action, as the results agree with the $\kappa$-expansion, in which there are no logarithms, and thus no poles in the drift. 

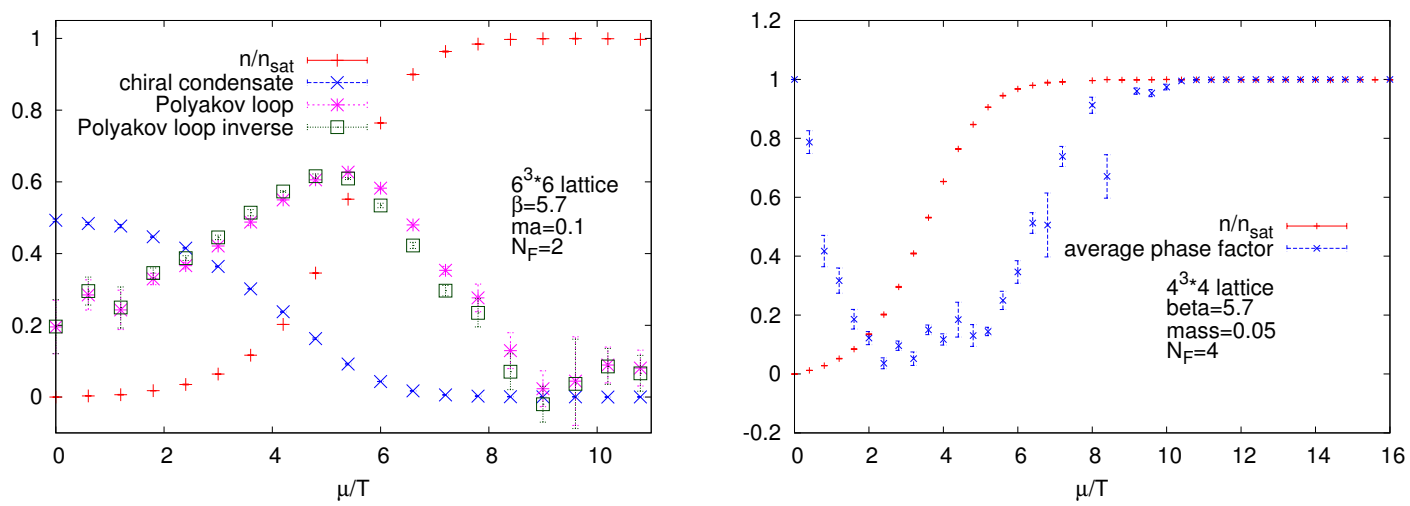

Figure 6: The fermionic density, the chiral condensate and the Polyakov loops in full QCD with 2 flavors of staggered fermions as a function of the chemical potential (left panel). The average phase factor defined in eq. (6.4) as a function of the chemical potential. (right panel, note that the lattice parameters are slightly different.)

\section{Results for full QCD}

The improvement in the theoretical understanding of the complex Langevin process and the introduction of the gauge cooling procedure has allowed for remarkable progress: the simulation of full QCD at light quark masses at high chemical potentials [6]. The drift term of the CLE includes a contribution from the fermionic determinant:

$$
K_{a x v}^{F}=\frac{N_{F}}{4} \operatorname{Tr}\left[M^{-1}(\mu, U) D_{a x v} M(\mu, U)\right],
$$

as written for the staggered formulation. This contribution is estimated using Gaussian noise vectors $\alpha$

$$
K_{a x v}^{F}=\frac{N_{F}}{4} \alpha^{+}\left(D_{a x v} M\right) M^{-1} \alpha,
$$

with $\left\langle\alpha_{x}^{*} \alpha_{y}\right\rangle=\delta_{x y}$. The inverse of the fermion matrix is calculated using the conjugate gradient algorithm, and this operation represents the main numerical cost of the simulation for small quark masses. So far unimproved staggered and Wilson fermions have been implemented in the CLE.

In Fig. 6 the behavior of various observables is shown as a function of the chemical potential for some fixed temperature above the deconfinement transition. Qualitatively the behavior is the same as in HDQCD. One sees the fermionic density rise until saturation is reached. The sign problem gets hard in the dense region before saturation, as shown in the right panel of Fig. 6 .

The spectrum of the staggered operator is shown in Fig. 7. The temperature is fixed at some value above the deconfinement transition, and the chemical potential is increased. For zero chemical potential the staggered operator is antihermitian with a real shift (the mass). At small chemical potentials the spectrum remains close to a mass shifted imaginary line, but the presence of $\mu$ starts to 'dilute' the spectrum also in real directions. As we increase the chemical potential further, the real part of the eigenvalues become significant. Nearing saturation (in the bottom right panel), the spectrum vacates the area around zero, as all excitations gain a large mass. Note that the distribution of the magnitude of the eigenvalues seems to have well defined lower limit, not allowing 

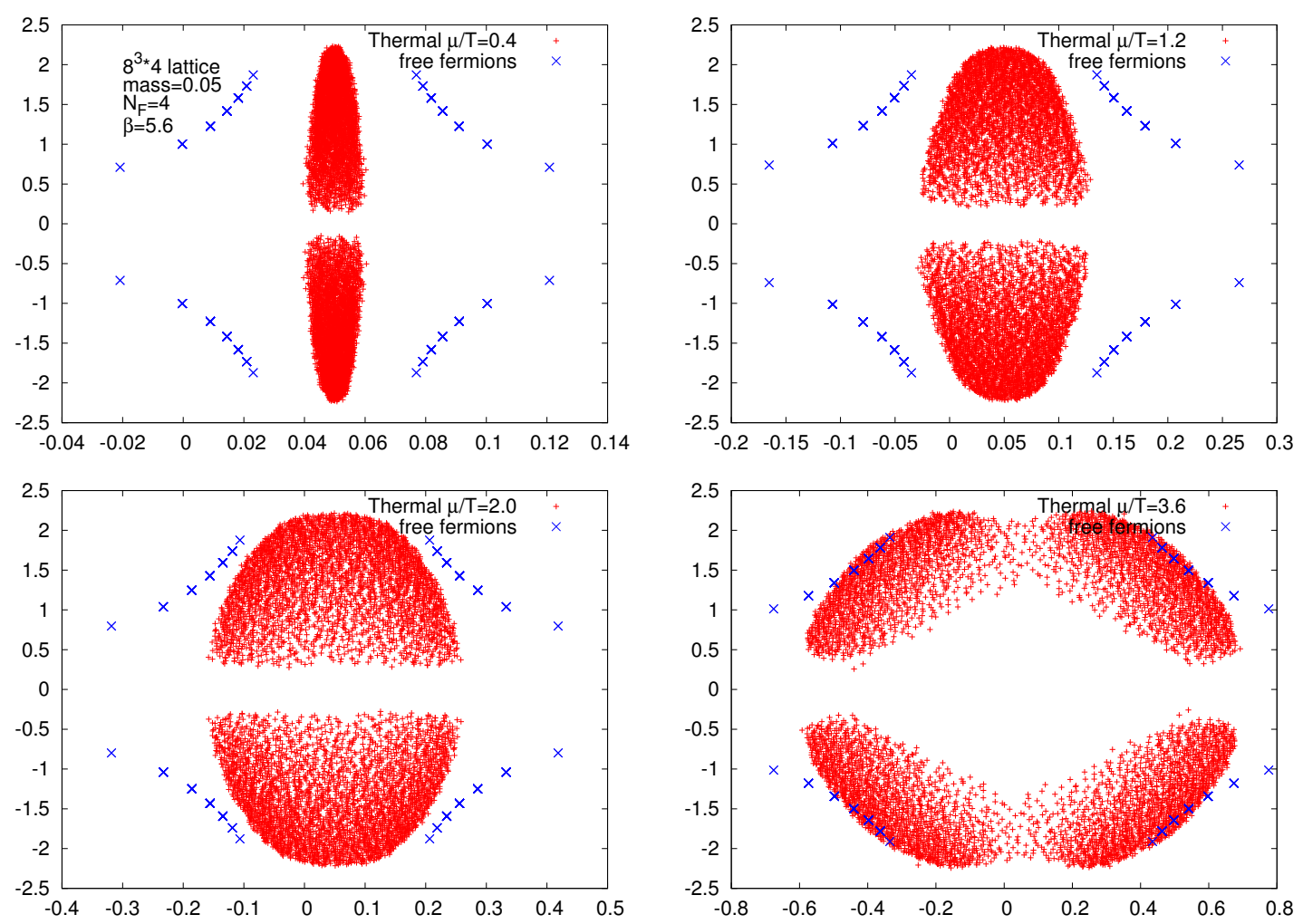

Figure 7: The spectrum of the staggered operator on the complex plane in a full simulation ('Thermal') as well as for a free fermionic theory at a fixed temperature for four different chemical potentials as indicated.

eigenvalues close to zero. At this high temperature this can be seen as a thermal contribution to the masses of excitations. This in consequence means that even if there are poles in the drift for certain gauge field configurations, the theory does not go close to these dangerous areas. This argument (as well as the argument from the expansion, spelled out in Sec. 7) tells us that at least at high temperatures the non-holomorphicity of the action causes no harm to the results.

These results show promise that one can use the CLE to map the phase diagram of full QCD. Many important checks are still to be done, such as the reproduction of the known behavior of the crossover line at small chemical potentials and comparison with (multi-parameter) reweighting methods [15].

\section{Conclusions}

I have reviewed two promising methods: the complex Langevin equation and the Lefschetz thimble, which evade the sign problem, thus provide a way to study dense systems (among others). Both methods work by extending the field manifold to a complexified manifold (in a slightly different way), and thus enable direct simulations of complex actions.

On the Lefschetz thimble, the dimensionality of the manifold is the same as the original real manifold, but the path is shifted onto the complex plane to such a path where the real part of the 
action is sharply peaked and the imaginary part is constant, thus the sign problem is absent (it is reintroduced in a mild way by the curvature of the manifold). In the complex Langevin method, the dimensionality of the field manifold is doubled, and it is sampled by the complex Langevin equation, which does not need interpretation of the action as a probability measure, thus there is no sign problem. The original theory is recovered by the expectation values.

The Lefschetz thimble approach has seen great interest in the past few years after its initial proposal, with many promising (numerical) results.

The complex Langevin method has a 'troubled past' in contrast. It was noticed more than 30 years ago that the method can solve complex action problems, but it was also noticed a few years later that in some cases it produces wrong results. Theoretical understanding of this issue has improved a lot in the recent years. This better understanding lead to the introduction of the gauge-cooling procedure which allows complex Langevin simulations of gauge theories. This in turn allowed the simulation of full QCD with light quarks at high chemical potentials for the first time.

Both methods still have a number of unanswered questions, but in my opinion they present the most promising means of solving the sign problem and exploring the phase diagram of full QCD for nonzero chemical potentials.

\section{References}

[1] P. de Forcrand, PoS LAT 2009 (2009) 010 [arXiv:1005.0539 [hep-lat]].

[2] G. Aarts, PoS LATTICE 2012 (2012) 017 [arXiv:1302.3028 [hep-lat]].

[3] G. Parisi, Phys. Lett. B 131 (1983) 393.

[4] G. Parisi and Y. s. Wu, Sci. Sin. 24 (1981) 483.

[5] E. Seiler, D. Sexty and I. -O. Stamatescu, Phys. Lett. B 723 (2013) 213 [arXiv:1211.3709 [hep-lat]].

[6] D. Sexty, Phys. Lett. B 729 (2014) 108 [arXiv:1307.7748 [hep-lat]].

[7] J. Berges and I.-O. Stamatescu, Phys. Rev. Lett. 95 (2005) 202003 [hep-lat/0508030]; J. Berges, S. Borsanyi, D. Sexty and I.-O. Stamatescu, Phys. Rev. D 75 (2007) 045007 [hep-lat/0609058]; J. Berges and D. Sexty, Nucl. Phys. B 799 (2008) 306 [arXiv:0708.0779 [hep-lat]].

[8] L. Bongiovanni, G. Aarts, E. Seiler, D. Sexty and I. O. Stamatescu, arXiv:1311.1056 [hep-lat] and in prep. ; L. Bongiovanni et. al. this proceedings.

[9] M. Cristoforetti et al. [AuroraScience Collaboration], Phys. Rev. D 86 (2012) 074506 [arXiv:1205.3996 [hep-lat]].

[10] G. Aarts, E. Seiler and I. -O. Stamatescu, Phys. Rev. D 81 (2010) 054508 [arXiv:0912.3360 [hep-lat]].

[11] A. Mollgaard and K. Splittorff, Phys. Rev. D 88 (2013) 116007 [arXiv:1309.4335 [hep-lat]].

[12] J. Greensite, arXiv:1406.4558 [hep-lat]. ; J. Greensite, this proceedings

[13] G. Aarts and F. A. James, JHEP 1201 (2012) 118 [arXiv:1112.4655 [hep-lat]].

[14] G. Aarts, F. A. James, J. M. Pawlowski, E. Seiler, D. Sexty and I. O. Stamatescu, JHEP 1303 (2013) 073 [arXiv:1212.5231 [hep-lat]].

[15] Z. Fodor, S.D. Katz, D. Sexty, in preparation. 
[16] G. Aarts, E. Seiler, D. Sexty and I. O. Stamatescu, arXiv:1408.3770 [hep-lat]; I. O. Stamatescu et. al., this proceedings.

[17] G. Aarts, L. Bongiovanni, E. Seiler, D. Sexty and I. -O. Stamatescu, Eur. Phys. J. A 49 (2013) 89 [arXiv:1303.6425 [hep-lat]].

[18] G. Aarts, L. Bongiovanni, E. Seiler, D. Sexty and I. -O. Stamatescu, arXiv:1310.7412 [hep-lat].

[19] G. Aarts, Phys. Rev. D 88 (2013) 9, 094501 [arXiv:1308.4811 [hep-lat]].

[20] G. Aarts, L. Bongiovanni, E. Seiler and D. Sexty, arXiv:1407.2090 [hep-lat], to appear in JHEP.

[21] M. Cristoforetti, F. Di Renzo, G. Eruzzi, A. Mukherjee, C. Schmidt, L. Scorzato and C. Torrero, Phys. Rev. D 89 (2014) 114505 [arXiv:1403.5637 [hep-lat]].

[22] A. Mukherjee, M. Cristoforetti and L. Scorzato, Phys. Rev. D 88 (2013) 5, 051502 [arXiv:1308.0233 [physics.comp-ph]].

[23] M. Cristoforetti, F. Di Renzo, A. Mukherjee and L. Scorzato, Phys. Rev. D 88 (2013) 5, 051501 [arXiv:1303.7204 [hep-lat]].

[24] A. Mukherjee and M. Cristoforetti, arXiv:1403.5680 [cond-mat.str-el].

[25] H. Fujii, D. Honda, M. Kato, Y. Kikukawa, S. Komatsu and T. Sano, JHEP 1310 (2013) 147 [arXiv:1309.4371 [hep-lat]].

[26] G. Aarts, Phys. Rev. Lett. 102 (2009) 131601 [arXiv:0810.2089 [hep-lat]].

[27] G. Eruzzi, this proceedings; F. Di Renzo, this proceedings.

[28] R. De Pietri, A. Feo, E. Seiler and I. -O. Stamatescu, Phys. Rev. D 76 (2007) 114501 [arXiv:0705.3420 [hep-lat]].

[29] T. D .Cohen, Phys. Rev. Lett. 91 (2003) 222001 [hep-ph/0307089].

[30] G. Aarts, F. Attanasio, B. Jaeger, E. Seiler, I.-O. Stamatescu and D. Sexty, in preparation; B. Jaeger et. al. this proceedings.

[31] G. Aarts and I. -O. Stamatescu, JHEP 0809 (2008) 018 [arXiv:0807.1597 [hep-lat]].

[32] I. Bender et. al. Nucl. Phys. Proc. Suppl. 26 (1992) 323.

[33] T. C. Blum, J. E. Hetrick and D. Toussaint, Phys. Rev. Lett. 76 (1996) 1019 [hep-lat/9509002].

[34] T. D. Bakeyev and P. de Forcrand, Phys. Rev. D 63 (2001) 054505 [hep-lat/0008006];

[35] G. Aarts, O. Kaczmarek, F. Karsch and I. -O. Stamatescu, Nucl. Phys. Proc. Suppl. 106 (2002) 456 [arXiv: hep-lat0110145].

[36] M. Fromm, J. Langelage, S. Lottini and O. Philipsen, JHEP 1201 (2012) 042 [arXiv:1111.4953 [hep-lat]].

[37] M. Fromm, J. Langelage, S. Lottini, M. Neuman and O. Philipsen, Phys. Rev. Lett. 110 (2013) 12, 122001 [arXiv:1207.3005 [hep-lat]].

[38] J. Greensite, J. C. Myers and K. Splittorff, Phys. Rev. D 88 (2013) 3, 031502 [arXiv:1306.3085 [hep-lat]].

[39] J. Langelage, M. Neuman and O. Philipsen, arXiv:1311.4409 [hep-lat].

[40] J. Langelage, M. Neuman and O. Philipsen, arXiv:1403.4162 [hep-lat]. 